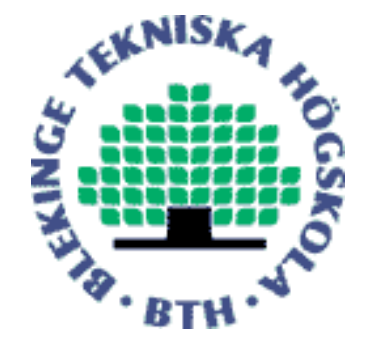

Copyright (C) 2010 IEEE.

Citation for the published paper:

Adjusted S-parametric Functions in the Creation of Symmetric Constraints

Elisabeth Rakus-Andersson

10th International Conference on Intelligent Systems Design and Applications - ISDA

2010 Cairo

This material is posted here with permission of the IEEE. Such permission of the IEEE does not in any way imply IEEE endorsement of any of BTH's products or services Internal or personal use of this material is permitted. However, permission to reprint/republish this material for advertising or promotional purposes or for creating new collective works for resale or redistribution must be obtained from the IEEE by sending a blank email message to pubs-permissions@iee.org.

By choosing to view this document, you agree to all provisions of the copyright laws protecting it. 


\title{
Adjusted $S$-parametric Functions in the Creation of Symmetric Constraints
}

\author{
Elisabeth Rakus-Andersson \\ Dept. of Mathematics and Science, School of Engineering \\ Blekinge Institute of Technology \\ S-37179 Karlskrona, Sweden \\ e-mail: Elisabeth.Andersson@bth.se
}

\begin{abstract}
One of the most important features of fuzzy set theory is its potential for the modeling of natural language expressions. Most works done on this topic focus on some parts of natural language, mostly those that correspond to the socalled "evaluating linguistic expressions". We build constraints for the mathematical substitutes of these expressions to mark characteristic limits on an ordered scale. In the current work we form families of constraints which originate from one function. By introducing a parameter in the initial membership function we can model the rest of family functions, whose shapes depend only on a number of functions and the length of a reference set. This procedure fits perfectly for being a segment of computer programs, where the loops providing us with many functions need only the initializations of two data values.
\end{abstract}

Keywords-linguistic variable; s-function; paramatric sfunction; parametric constraints of fuzzy sets

\section{INTRODUCTION}

In some works devoted to the creation of contents of linguistic lists $[1,2,3,4,5,6]$ the membership functions of fuzzy sets representing the list expressions are derived rather intuitively by applying symmetry for opposite atomic expressions. The borders of fuzzy sets were decided in advance without using any formal criteria. This method was used in the papers composed by Adlassnig [1, 2], in which the diagnosis process based on clinical symptoms was the item of investigations. The number of functions was predetermined as well as their borders, which made the model difficult to be quickly changeable. The use of parametric modifiers, inserted in the restrictions, appeared for linear membership functions in the work by BouchonMeunier [7] and, for the second degree polynomials, in the paper by Novák and Perfilieva [8].

Since the parametric expressions rearrange one function to several forms attached to a linguistic list we wish to test different effects of the parameter actions as results of our own original investigations. As the basic function to be modified we have selected the $s$-class function. The linguistic lists are necessary to develop in such systems as fuzzy control or medical diagnosing where the verbal list is the only source of communication with some specialists on the stage of arranging logical rules of the methods. We fuzzify the terms of lists to either defuzzify these fuzzy sets or to perform the operations on them. The general parametric form of membership functions allows creating arbitrary lists without any intuitive assumptions.

\section{MEMBERSHIP FUNCTIONS OF THE "LEFTMOST" FAMILY OF FUZZY SETS}

Let us suppose that the linguistic list is constructed as a sampling of terms $F_{1}, F_{2}, \ldots, F_{n}$, where $n$ is an odd positive integer greater or equal to 5 . Each term is a name of the corresponding fuzzy set, whose restriction is supposed to be created as the common formula depending on the $i^{\text {th }}$ value, where $i=1, \ldots, n$. We assume that supports of restrictions $\mu_{F_{i}}(x), i=1,2, \ldots, n$, will cover parts of the reference set $A$ $=[0, E]$, where $E$ is a real number.

We divide all expressions $F_{i}$ in three groups, namely, a family of "leftmost" sets $F_{1}, \ldots, F_{\frac{n-1}{2}}$, the set $F_{\frac{n+1}{2}}$ "in the middle" and a collection of "rightmost" sets $F_{\frac{n+3}{2}}, \ldots, F_{n}$.

To design the membership functions of $F_{i}$ the $s$-class function

$$
s(x, \alpha, \beta, \gamma)=\left\{\begin{array}{lll}
0 & \text { for } \quad x \leq \alpha, \\
2\left(\frac{x-\alpha}{\gamma-\alpha}\right)^{2} & \text { for } \quad \alpha \leq x \leq \beta, \\
1-2\left(\frac{x-\gamma}{\gamma-\alpha}\right)^{2} & \text { for } \beta \leq x \leq \gamma, \\
1 & \text { for } x \geq \gamma,
\end{array}\right.
$$

will be adopted. The point $(\alpha, 0)$ starts the graph of the $s$ function whereas the point $(\gamma, 1)$ terminates this graph. The parameter $\beta$ is computed as the arithmetic mean of $\alpha$ and $\gamma$. In $x=\beta$ the $s$-function reaches the value of 0.5 .

Let us first design the parameters of the membership function characteristic of the "leftmost" family. To preserve the equal distances between the adjacent functions and warrant their symmetrical patterns we measure the distances on the level of membership degrees equaling 0.5 . If we suppose that the last "leftmost" function should take the value of 0.5 in $x=\frac{E}{2}$, then we will find the breadth of each "leftmost" function as $\frac{E}{2}$ divided by the number of leftmost functions given as $\frac{n-1}{2}$, i.e., the breadth is evaluated as $\frac{E}{n-1}$ on the level of membership degree 0.5. Particularly, we can 
assume that the $\beta$-value of the first leftmost function $F_{1}$ is set as $\beta_{F_{1}}=\frac{E}{n-1}$.

To determine $\alpha_{F_{1}}$ we subtract the half of $\beta_{F_{1}}$ from $\beta_{F_{1}}$, i.e., $\alpha_{F_{1}}=\frac{E}{n-1}-\frac{E}{2(n-1)}=\frac{E}{2(n-1)}$.

The value of $\gamma_{F_{1}}$ is created by the addition of the half of $\beta_{F_{1}}$ to $\beta_{F_{1}}$. We thus state $\gamma_{F_{1}}=\frac{E}{n-1}+\frac{E}{2(n-1)}=\frac{3 E}{2(n-1)}$. Since the beginning of $F_{1}$ is planned to be placed in $(0,1)$ then $\mu_{F_{1}}(x)=1-s\left(x, \frac{E}{2(n-1)}, \frac{E}{n-1}, \frac{3 E}{2(n-1)}\right)$.

The membership function of $F_{1}$ is thus expanded as

$$
\mu_{F_{1}}(x)=\left\{\begin{array}{lll}
1 & \text { if } \quad x \leq \frac{E}{2(n-1)}, \\
1-2\left(\frac{x-\frac{E}{2(n-1)}}{\frac{E}{n-1}}\right)^{2} & \text { if } \quad \frac{E}{2(n-1)} \leq x \leq \frac{E}{n-1}, \\
2\left(\frac{x-\frac{3 E}{2(n-1)}}{\frac{E}{n-1}}\right)^{2} & \text { if } \quad \frac{E}{n-1} \leq x \leq \frac{3 E}{2(n-1)}, \\
0 & \text { if } \quad x \geq \frac{3 E}{2(n-1)} .
\end{array}\right.
$$

Suppose that $n=11$ and $E=100$. The membership function of $F_{1}$ is then rearranged to

$$
\mu_{F_{1}}(x)=\left\{\begin{array}{lll}
1 & \text { if } \quad x \leq 5 \\
1-2\left(\frac{x-5}{10}\right)^{2} & \text { if } \quad 5 \leq x \leq 10 \\
2\left(\frac{x-15}{10}\right)^{2} & \text { if } \quad 10 \leq x \leq 15 \\
0 & \text { if } \quad x \geq 15
\end{array}\right.
$$

to be further depicted in Fig. 1.

All constraints characteristic of the "leftmost" family of fuzzy sets will be derived by inserting of parameter $k, k=$ $1, \ldots, \frac{n-1}{2}$, in (2) due to $[10,11,12,13,14]$ to expand (2) as

$$
\begin{aligned}
& \mu_{F_{k}}(x)= \\
& \left\{\begin{array}{l}
1 \text { if } x \leq \frac{E}{2(n-1)}+(k-1) \frac{E}{n-1}, \\
1-2\left(\frac{x-\left(\frac{E}{2(n-1)}+(k-1) \frac{E}{n-1}\right)}{\frac{E}{n-1}}\right)^{2} \text { if } \\
\frac{E}{2(n-1)}+(k-1) \frac{E}{n-1} \leq x \leq \frac{E}{n-1}+(k-1) \frac{E}{n-1}, \\
2\left(\frac{x-\left(\frac{3 E}{2(n-1)}+(k-1) \frac{E}{n-1}\right)}{\frac{E}{n-1}}\right)^{2} \text { if } \\
\frac{E}{n-1}+(k-1) \frac{E}{n-1} \leq x \leq \frac{3 E}{2(n-1)}+(k-1) \frac{E}{n-1}, \\
0 \text { if } x \geq \frac{3 E}{2(n-1)}+(k-1) \frac{E}{n-1} .
\end{array}\right.
\end{aligned}
$$

Formula (4) has been constructed in conformity with the intension that the second leftmost function $F_{2}$ should be removed forward along the $x$-axis. The distance $d\left(F_{1}, F_{2}\right)$ ought to be equal to $\frac{E}{n-1}$ when measuring it between $\left(\beta_{F_{1}}, 0.5\right)$ and $\left(\beta_{F_{2}}, 0.5\right)$.

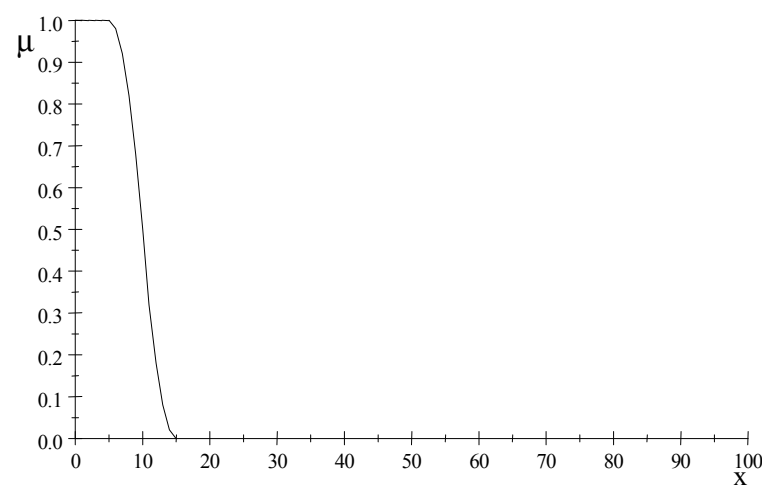

Figure 1. The membership function of $F_{1}$

The intervals of the $F_{2}$ membership function have to be changed to the new allocation and, to warrant this effect, the length of $\frac{E}{n-1}$ is added to the interval borders of the $F_{1}$ function. Moreover, the same length $\frac{E}{n-1}$ must be added to $\alpha_{F_{1}}$ and $\gamma_{F_{1}}$ in the numerator of (2) to get the full effect of the replacement of $F_{1}$ to the position of $F_{2}$.

The same procedure is repeated for the membership function of $F_{3}$, which means the inserting of two lengths of $\frac{E}{n-1}$ in (4) to maintain the right position of $F_{3}$ when comparing to $F_{1}$. Generally, we add the lengths of $(k-1) \frac{E}{n-1}$ to the parameters and borders of $F_{1}, k=1$, $2, \ldots, \frac{n-1}{2}$, to initiate all membership functions of the "leftmost" collection of fuzzy sets. Factor $k-1$ is adjusted to generate even $F_{1}$ by using formula (4), which is common for all leftmost functions. The membership functions obtained by (4) are sketched in Fig. 2.

\section{CONSTRAINTS OF THE "RIGHTMOST" FAMILY OF FUZZY SETS}

The last function $\mu_{F_{n}}(x)$ should possess $F_{1}$ 's inverted shape.

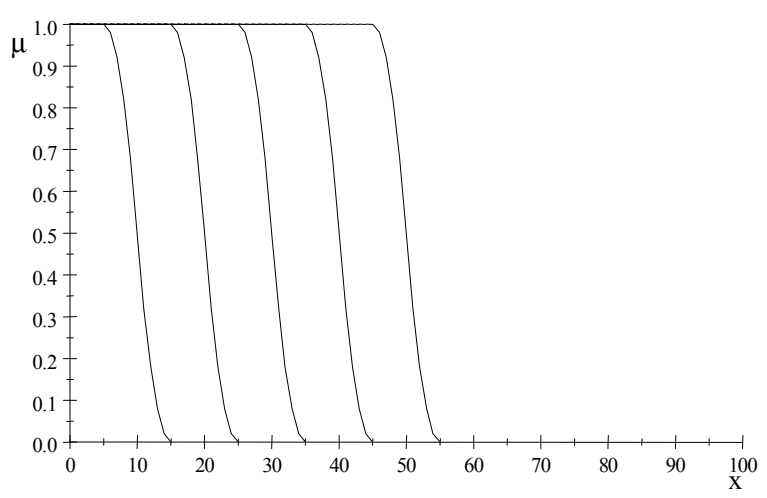

Figure 2. The constraints of $F_{1}, \ldots, F_{\frac{n-1}{2}}$ 
We generate the membership function of $F_{n}$ by

$$
\begin{aligned}
& \mu_{F_{n}}(x)= \\
& \left\{\begin{array}{lll}
0 & \text { if } \quad x \leq E-\frac{3 E}{2(n-1)}, \\
2\left(\frac{x-\left(E-\frac{3 E}{2(n-1)}\right)}{\frac{E}{n-1}}\right)^{2} & \text { if } \quad & E-\frac{3 E}{2(n-1)} \leq x \leq E-\frac{E}{n-1}, \\
1-2\left(\frac{x-\left(E-\frac{E}{2(n-1)}\right)}{\frac{E}{n-1}}\right)^{2} & \text { if } & E-\frac{E}{n-1} \leq x \leq E-\frac{E}{2(n-1)}, \\
1 & \text { if } \quad x \geq E-\frac{E}{2(n-1)} .
\end{array}\right.
\end{aligned}
$$

$F_{n}$ is symmetric to $F_{1}$ over interval $[0, E]$; therefore $\mu_{F_{1}}(x)=1-s\left(x, \frac{E}{2(n-1)}, \frac{E}{n-1}, \frac{3 E}{2(n-1)}\right)$ will be converted to $\mu_{F_{n}}(x)=s\left(x, E-\frac{3 E}{2(n-1)}, E-\frac{E}{n-1}, E-\frac{E}{2(n-1)}\right)$. We draw $F_{n}$ 's membership function in Fig. 3.

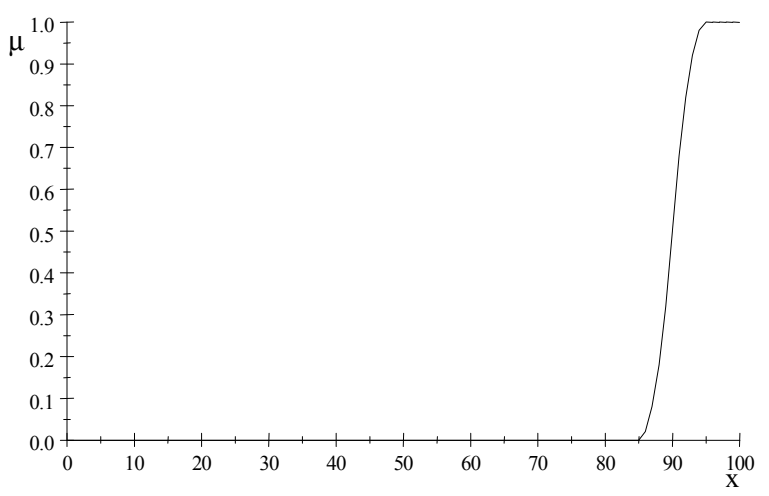

Figure 3. The constraint of $F_{n}$

We can now obtain the "rightmost" family of sets when moving the membership function of $F_{n}$ backward to the positions of $F_{n-1}, F_{n-2}, \ldots, F_{\frac{n+3}{2}}$. The common formula for the "rightmost" family of functions is proposed as

$$
\begin{aligned}
& \mu_{F_{n-k+1}}(x)= \\
& \left\{\begin{array}{l}
0 \text { if } x \leq E-\left(\frac{3 E}{2(n-1)}+(k-1) \frac{E}{n-1}\right), \\
2\left(\frac{x-\left(E-\left(\frac{3 E}{2(n-1)}+(k-1) \frac{E}{n-1}\right)\right)}{\frac{E}{n-1}}\right)^{2} \text { if } \\
E-\left(\frac{3 E}{2(n-1)}+(k-1) \frac{E}{n-1}\right) \leq x \leq E-\left(\frac{E}{n-1}+(k-1) \frac{E}{n-1}\right), \\
1-2\left(\frac{x-\left(E-\left(\frac{E}{2(n-1)}+(k-1) \frac{E}{n-1}\right)\right.}{\frac{E}{n-1}}\right)^{2} \text { if } \\
-\left(\frac{E}{n-1}+(k-1) \frac{E}{n-1}\right) \leq x \leq E-\left(\frac{E}{2(n-1)}+(k-1) \frac{E}{n-1}\right), \\
1 \text { if } x \geq E-\left(\frac{E}{2(n-1)}+(k-1) \frac{E}{n-1}\right)
\end{array}\right.
\end{aligned}
$$

for $k=1, \ldots, \frac{n-1}{2}$.

Fig. 4 contains the graphs of all "rightmost" functions for $n=11$ and $E=100$.

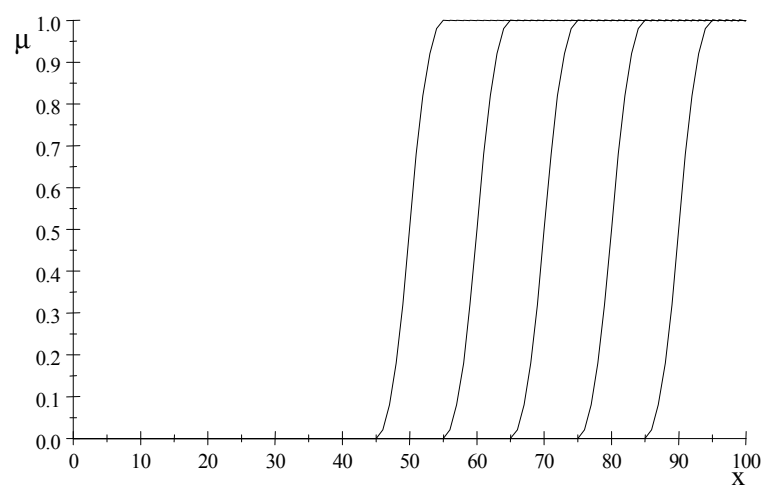

Figure 4. The constraints of $F_{\frac{n+3}{2}}, \ldots, F_{n}$

\section{A Restriction OF THE “IN THE MiddLE” FuZZy Set}

The function of $F_{\frac{n+1}{2}}$ is constructed as a $\pi$-function

$$
\pi(x)=\left\{\begin{array}{lll}
s\left(x, \alpha_{1}, \beta_{1}, \gamma_{1}=\alpha_{2}\right) & \text { if } \quad x \leq \gamma_{1}=\alpha_{2}, \\
1-s\left(x, \alpha_{2}=\gamma_{1}, \beta_{2}, \gamma_{2}\right) & \text { if } \quad x \geq \gamma_{1}=\alpha_{2} .
\end{array}\right.
$$

For (7) the distance between $\left(\beta_{1}, 0.5\right)$ and $\left(\gamma_{1}, 0.5\right)$ is measured as $\frac{E}{n-1}$. The value of $\beta_{1, F_{\frac{n+1}{2}}}$ will be thus equal to $\frac{E}{2}-\frac{E}{n-1}=\frac{E(n-3)}{2(n-1)}$. To compute $\alpha_{1, F_{\frac{n+1}{2}}}$ we subtract two lengths $\frac{E}{n-1}$ from $\frac{E}{2}$, which yields $\frac{E}{2}-\frac{2 E}{n-1}=\frac{E(n-5)}{2(n-1)}$.

The values of $\gamma_{1}$ and $\alpha_{2}$ are stated as $\frac{E}{2}$.

To determine the parameters of the right $s$ function of $F_{\frac{n+1}{2}}$ we add the distances $\frac{E}{n-1}$ and $\frac{2 E}{n-1}$ to $\frac{E}{2}$. Hence, $\beta_{2, F_{\frac{n+1}{2}}}=\frac{E(n+1)}{2(n-1)}$ whereas $\gamma_{2, F_{\frac{n+1}{2}}}=\frac{E(n+3)}{2(n-1)}$.

The membership function of $F_{\frac{n+1}{2}}$ is finally given by

$$
\mu_{F_{\frac{n+1}{2}}}(x)=\left\{\begin{array}{lll}
0 & \text { if } \quad x \leq \frac{E(n-5)}{2(n-1)}, \\
2\left(\frac{x-\frac{E(n-5)}{2(n-1)}}{\frac{2 E}{n-1}}\right)^{2} & \text { if } & \frac{E(n-5)}{2(n-1)} \leq x \leq \frac{E(n-3)}{2(n-1)}, \\
1-2\left(\frac{x-\frac{E}{2}}{\frac{2 E}{n-1}}\right)^{2} & \text { if } & \frac{E(n-3)}{2(n-1)} \leq x \leq \frac{E}{2}, \\
1-2\left(\frac{x-\frac{E}{2}}{\frac{2 E}{n-1}}\right)^{2} & \text { if } & \frac{E}{2} \leq x \leq \frac{E(n+1)}{2(n-1)}, \\
2\left(\frac{x-\frac{E(n+3)}{2(n-1)}}{\frac{2 E}{n-1}}\right)^{2} & \text { if } & \frac{E(n+1)}{2(n-1)} \leq x \leq \frac{E(n+3)}{2(n-1)}, \\
0 & \text { if } & x \geq \frac{E(n+3)}{2(n-1)}
\end{array}\right.
$$

and, for $n=11$ and $E=100$, plotted in Fig. 5 . 


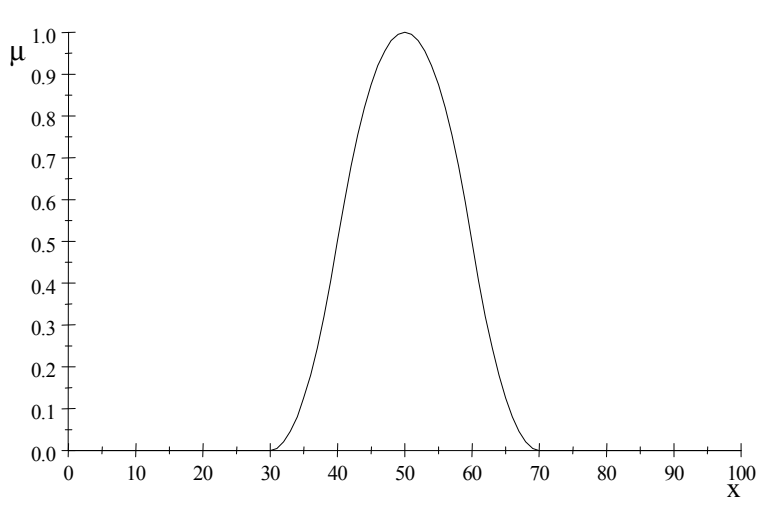

Figure 5. The membership function of $F_{\frac{n+1}{2}}$

All functions $F_{1}, \ldots, F_{n}$ are placed in Fig. 6 when setting $n$ $=11$ and $E=100$ in (4), (6) and (8).

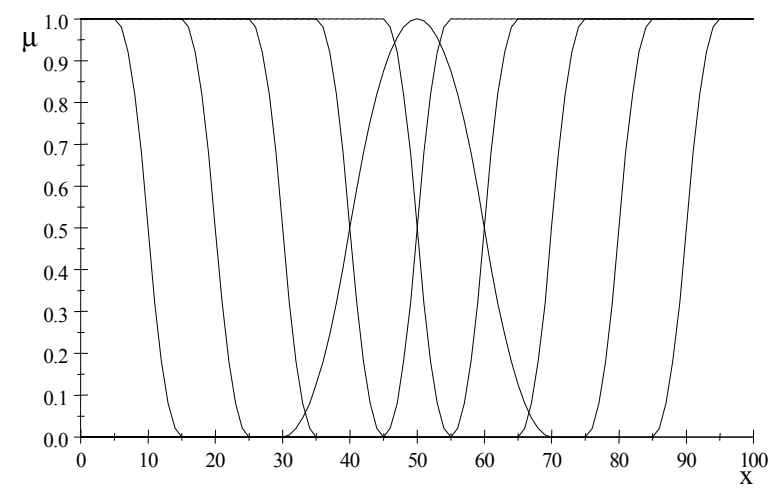

Figure 6. The membership functions of $F_{1}, \ldots, F_{n}$

\section{Example 1}

To be able to demonstrate the partial results of the algorithm in the form of drawings we have earlier assumed $n=11$ and $E=100$. These quantities can assist a list "symptom presence in diagnosis" defined in a reference set $[0,100]$.

The terms of the list are established as

"presence" $=\left\{F_{1}=\right.$ "never", $F_{2}=$ "almost never", $F_{3}=$ "very seldom", $F_{4}=$ "seldom", $F_{5}=$ "rather seldom", $F_{6}=$ "moderately", $F_{7}=$ "rather often", $F_{8}=$ "often", $F_{9}=$ "very often", $F_{10}=$ "almost always", $F_{11}=$ "always"\}.

The list "presence" constitutes a dominant factor in the problem of deciding medical diagnosis [1, 2, 9]. The fuzzy sets constructed due to the algorithm can be defuzzified or included in some operations of the diagnosing paradigm. We refer a reader to [9] to make a closer acquaintance with the descriptions of linguistic variables in diagnostic process.

\section{THE Block Scheme of THE AlgORITHM}

All steps of the discussed algorithm, which initiates three sets of membership functions corresponding to a list of terms, can be sampled in the block scheme. We need to follow the steps of the scheme together with formulas (4), (6) and (8) to write a computer program. We emphasize that the only data, used in the algorithm, are the length of the reference set and the number of functions. We do not need to specify the sets' borders in the process of the program initialization, as most of programmers do since the borders are computed automatically by formulas (4), (6) and (8).

The steps of the algorithm block scheme are sampled in Fig. 7.

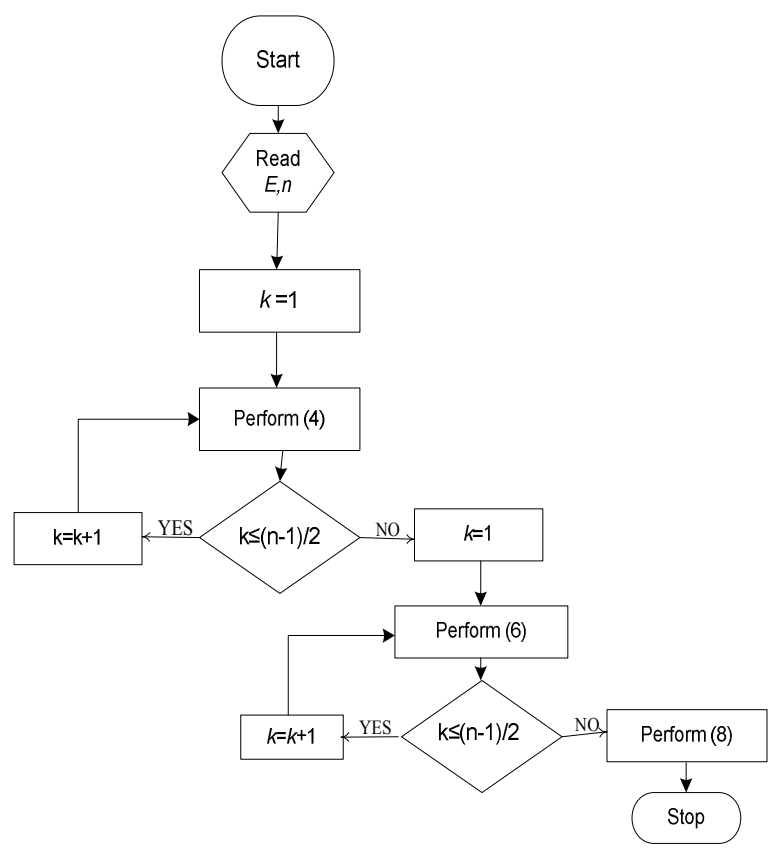

Figure 7. The block scheme of the procedure implementing membership functions of $F_{1}, \ldots, F$

\section{Example 2}

To approximate the survival length in stomach cancer patients the fuzzy control algorithm, primary developed by Mamdani and Assilian [15], has been adopted. For two biological markers $X=$ "age" and $Y=$ "CRP-value" we have designed two lists in order to divide $X$ and $Y$ in intensity levels. $X$ is differentiated as

$X=$ "age" $=\left\{X_{1}=\right.$ "very young", $X_{2}=$ "young", $X_{3}=$ "middle-aged", $X_{4}=$ "old", $X_{5}=$ "very old" over $[0,100]$.

The membership functions of $X_{1}, X_{2}$, belonging to the "leftmost" family, are derived as

$$
\mu_{X_{k}}(x)=\left\{\begin{array}{l}
1 \text { if } x \leq 12.5+(k-1) 25 \\
1-2\left(\frac{x-(12.5+(k-1) 25)}{25}\right)^{2} \text { if } \\
12.5+(k-1) 25 \leq x \leq 25+(k-1) 25 \\
2\left(\frac{x-(37.5+(k-1) 25)}{25}\right)^{2} \text { if } \\
25+(k-1) 25 \leq x \leq 37.5+(k-1) 25 \\
0 \text { if } x \geq 37.5+(k-1) 25
\end{array}\right.
$$


for $k=1,2$.

The "in the middle" set $X_{3}$ is given by

$$
\mu_{X_{3}}(x)=\left\{\begin{array}{lll}
0 & \text { if } \quad x \leq 0 \\
2\left(\frac{x-0}{50}\right)^{2} & \text { if } \quad 0 \leq x \leq 25 \\
1-2\left(\frac{x-50}{50}\right)^{2} & \text { if } \quad 25 \leq x \leq 50 \\
1-2\left(\frac{x-50}{50}\right)^{2} & \text { if } \quad 50 \leq x \leq 75 \\
2\left(\frac{x-100}{50}\right)^{2} & \text { if } \quad 75 \leq x \leq 100 \\
0 & \text { if } \quad x \geq 100
\end{array}\right.
$$

by

Lastly, the "rightmost" sets $X_{4}$ and $X_{5}$ are characterized

$$
\begin{aligned}
& \mu_{X_{5-k+1}}(x)= \\
& \left\{\begin{array}{l}
0 \text { if } x \leq 100-(37.5+(k-1) 25), \\
2\left(\frac{x-(100-(37.5+(k-1) 25))}{25}\right)^{2} \text { if } \\
100-(37.5+(k-1) 25) \leq x \leq 100-(25+(k-1) 25), \\
1-2\left(\frac{x-(100-(12.5+(k-1) 25))}{25}\right)^{2} \text { if } \\
100-(25+(k-1) 25) \leq x \leq 100-(12.5+(k-1) 25), \\
1 \text { if } x \geq 100-(12.5+(k-1) 25),
\end{array}\right.
\end{aligned}
$$

where $k=1,2$.

Fig. 8 collects all curves assisting $X_{1}, \ldots, X_{5}$.

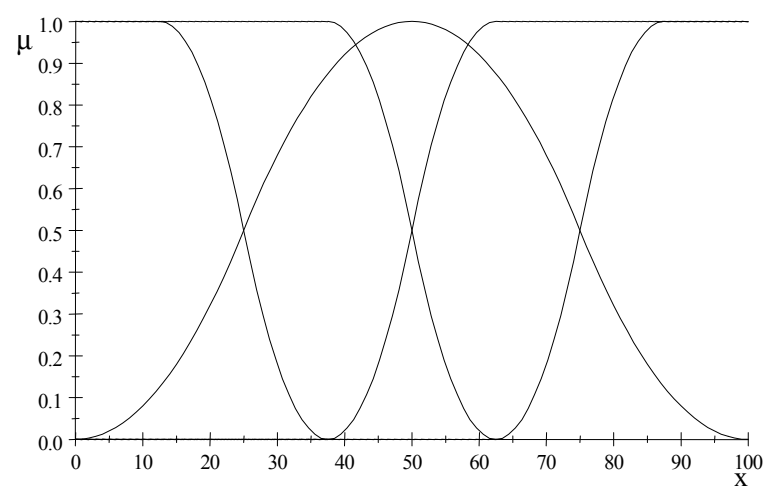

Figure 8. Membership functions of $X_{1}, \ldots, X_{5}$

The second independent variable $Y$ in fuzzy control model is divided into intensity levels

$Y=$ "CRP-value" $=\left\{Y_{1}=\right.$ "normal", $Y_{2}=$ "almost normal", $Y_{3}=$ "very little heightened", $Y_{4}=$ "little heightened", $Y_{5}=$ "rather little heightened", $Y_{6}=$ "moderately heightened", $Y_{7}$ $=$ "rather high", $Y_{8}=$ "high", $Y_{9}=$ "very high", $Y_{10}=$ "almost critically high", $Y_{11}=$ "critically high" $\}$. over $[0,100]$.

We can accept functions presented by Fig. 6 as the membership functions of $Y_{1}, \ldots, Y_{n}(n=11, E=100)$.
We cannot discuss the whole procedure of fuzzy control action in the current paper for the reason of the lack of space. Let us only note how easy is to perform the first steps of the control algorithm by using the functions $F_{1}, \ldots, F_{n}$.

Suppose that $x=77$.

The value of $x$ belongs to $X_{5}$ since, for $k=1$, interval $100-(25+(k-1) 25) \leq x=77 \leq 100-(12.5+(k-1) 25)$

becomes equivalent to $75 \leq x=77 \leq 87.5$.

The associated membership degree is equal to

$\mu_{X_{5, k=1}}(77)=1-2\left(\frac{77-(100-(12.5+(k-1) 25))}{25}\right)^{2}=1-2\left(\frac{77-(100-12.5)}{25}\right)^{2}$

$=0.647$.

The same value $x=77$ is also a member of $X_{4}$ for $k=2$ with the membership $\mu_{X_{5, k=2}}(77)=1$ because of the relation $x=77 \geq 100-(12.5+(k-1) 25)$ stated as $x=77 \geq 62.5$.

Integer $x=77$ is even found in set $X_{3}$ due to its presence in interval $75 \leq x \leq 100$. The assisting membership degree obtains the value of $\mu_{X_{3}}(77)=2\left(\frac{77-100}{50}\right)^{2}=0.423$.

For the associated value of $y$ from pair $(x, y)$ we should find all membership degrees in these sets from the $Y$-list, which include $y$ in their supports.

By following the Mamdani controller [15] we use the expert system to find the logical rules of the type IF "level of $x$ " and "level of $y$ " THEN "level of survival" to assimilate sets containing $x$ and $y$ with one of survival levels, which can also be extracted due to the proposed algorithm from Fig. 7. We follow next steps of Mamdani's rules to use in them the combinations of membership degrees computed in accordance with (4), (6) and (8). The common consequence set, based on partial results corresponding to each logical rule, will be defuzzified to provide us with a distinct value of survival. Both the earlier designed membership functions and the minimal membership degrees, concatenated for $x$ and $y$, constitute the crucial factors of the rules.

For each pair $(x, y), x \in X, y \in Y$, we can easily calculate the values of membership degrees in different fuzzy sets by extracting the appropriate $k$-numbers from the set list. This technique should simplify computations by depriving the model of a large amount of membership functions designed one by one.

\section{CONCLUSIONS}

In many algorithms the borders of membership functions, representing a certain list, are introduced as the initial data. We have used the pattern of the $s$-functions to model constrains of fuzzy sets without their predetermined start points and endpoints. For a large amount of functions a computer program is recommended to produce a uniform and symmetric shape of fuzzy restrictions. Due to the block scheme proposed, which constitutes the original model created by the author, we can easily convert the scheme to a sequence of program commands. We emphasize that the 
only data, demanded by the model are restricted to the number of functions and the length of the reference set.

We hope that all transformations performed on the membership functions have demonstrated the logical and elegant design of the mathematical scenario, which should be regarded as an advantage of the presented model.

\section{ACKNOWLEDGMENT}

The author thanks the Blekinge Research Board for the grant funding the current research.

\section{REFERENCES}

[1] K. P. Adlassnig, "A Fuzzy Logical Model of Computer-assisted Medical Diagnosis," Methods of Information in Medicine 19, $\mathrm{nr} 3$, 1980, pp. 141-148.

[2] K. P. Adlassnig, "Fuzzy Modeling and Reasoning in a Medical Diagnostic Expert System," EVD in Medizin und Biologie 17, 1/2, 1986, pp. 12-20.

[3] L. A. Zadeh, Calculus of Fuzzy Restrictions, Fuzzy Sets and Their Applications to Cognitive and Decision Processes, Academic Press, London, 1975.

[4] L. A. Zadeh, "A Computational Approach to Fuzzy Quantifiers in Natural Languages," Computers and Mathematics 9, 1983, pp. 149184.

[5] L. A. Zadeh, "Test-score Semantics as a Basis for a Computational Approach to the Representation of Meaning," Literary and Linguistic Computing 1, 1986, pp. 24-35.

[6] L. A. Zadeh, "From Computing with Numbers to Computing with Words - From Manipulation of Measurements to Manipulation of Perceptions," IEEE Transactions on Circuits and Systems 45, 1999, pp. 105-119.

[7] B. Bouchon-Meunier, "Fuzzy Logic and Knowledge Representation Using Linguistic Modifiers," in Fuzzy Logic for the Management of Uncertainty, Edited by L. A. Zadeh and J. Kacprzyk, Wiley, New York, 1992.

[8] V. Novák and I. Perfilieva, "Evaluating of Linguistic Expressions and Functional Fuzzy Theories in Fuzzy Logic," in Computing with Words in Information - Intelligent Systems 2, Edited by L. A. Zadeh and J. Kacprzyk, Studies in Fuzziness and Soft Computing Series, vol. 33, Springer-verlag, Berlin Heidelberg New York, 1999, pp. 383-406.

[9] E. Rakus-Andersson, Fuzzy and Rough Techniques in Medical Diagnosis and Medication, Springer-verlag, Berlin Heidelberg, 2007.

[10] E. Rakus-Andersson and L. Jain, "Computational Intelligence in Medical Decisions Making," In Recent Advances in Decision Making, Edited by E. Rakus-Andersson, R. R. Yager and L. Jain, Series: Studies of Computational Intelligence, Springer, Berlin Heidelberg, 2009, pp. 145-159.

[11] E. Rakus-Andersson, "Approximate Reasoning in Surgical Decisions," In Proceedings of the International Fuzzy Systems Association World Congress - IFSA 2009, Instituto Superior Technico, 2009, 225-230.

[12] E. Rakus-Andersson, "One-dimensional Model of Approximate Reasoning in Surgical Considerations," in print in Intuitionistic Fuzzy Sets and Generalized Nets, Publishing House of Polish Academy of Sciences - EXIT, Warsaw, 2010.

[13] E. Rakus Andersson, Fuzzy Control, Intern Research Report, 2010.

[14] E. Rakus-Andersson, H. Zettervall and M. Erman, "Prioritization of Weighted Strategies in the Multi-player Games with Fuzzy Entries of the Payoff Matrix,” Int. J. of General Systems, Vol, 39, Issue 3, 2010, pp. 291-304.
[15] E. H. Mamdani and S. Assilian, "An Experiment in Linguistic Synthesis with a Fuzzy Logic Controller," Int. J. Man-Machine Studies 7, 1973, pp. 1-13. 